\title{
Joint Optimal Pricing and Ordering Decisions for Seasonal Products with Weather-Sensitive Demand
}

\author{
Hongyong Fu, ${ }^{1}$ Bin Dan, ${ }^{2,3}$ and Xiangkai Sun ${ }^{4}$ \\ ${ }^{1}$ School of Management, Southwest University of Political Science and Law, Chongqing 401120, China \\ ${ }^{2}$ School of Economics and Business Administration, Chongqing University, Chongqing 400044, China \\ ${ }^{3}$ Chongqing Key Laboratory of Logistics, Chongqing University, Chongqing 400030, China \\ ${ }^{4}$ College of Mathematics and Statistics, Chongqing Technology and Business University, Chongqing 400067, China \\ Correspondence should be addressed to Bin Dan; danbin@cqu.edu.cn
}

Received 30 November 2013; Accepted 22 February 2014; Published 27 March 2014

Academic Editor: Leonid Shaikhet

Copyright (c) 2014 Hongyong Fu et al. This is an open access article distributed under the Creative Commons Attribution License, which permits unrestricted use, distribution, and reproduction in any medium, provided the original work is properly cited.

\begin{abstract}
Retailers selling seasonal products often face the challenge in matching their inventory levels with uncertain market demand which is sensitive to weather conditions, such as the average seasonal temperature. Therefore, how to make the joint ordering and pricing decisions may help retailers to increase their profits. In this paper, we address the joint determination of pricing and ordering decisions in a newsvendor setting, where a retailer (newsvendor) sells the seasonal products and faces demand risk due to weather uncertainty. We show that the maximum expected profit function is continuous and concave, so the optimal solution to the retail price and order quantity exists and it is the one and only solution. In addition, we numerically investigate the impacts of related parameters on the retailer's expected profit and the optimal pricing and ordering decisions and illustrate some useful management insights into the economic behavior of firms.
\end{abstract}

\section{Introduction}

Non-catastrophic (examples of catastrophic weather conditions mainly include natural disaster type weathers, such as blizzards, tornados, and cyclonic) weather represents an important determinant of demand for many seasonal products such as weatherproof, soft drinks and ice cream products, coats and sweaters, and heating oil. The U.S. National Research Council has estimated that $46 \%$ of U.S. gross domestic product (GDP) is affected by weather [1]. Against the backdrop, this paper is concerned with uncertainty demand risk that business firms may have to face under non-catastrophic weather conditions.

Weather affects the companies' economic activities in many business industries. In the retail industry, Wal-Mart Stores, Inc., reported in June 2005 that its inventory levels were higher than normal because below-normal temperatures crimped demand [2]. In the North of England, a jacket firm reported a third quarter drop in earnings of $12 \%$ compared with the third-quarter of previous year. The firm believed that the losses were due to milder than usual winter [3]. Demand for propane heating oil is also weather sensitive. If the season is warmer than normal then the sales volume of propane heating oil will decrease, which leaves the propane heating oil distributor with excess inventory and the associated management expenses [4]. USA Today reported that warm weather in December 2006 in the northeastern part of the United States caused a dramatic fall-off in the demand for coats and sweaters, and subsequently, a cold April in 2007 had the same effect on springtime clothing [5]. In addition, historical data indicates a $91 \%$ correlation between the temperature and the number of items of cold weather clothing sold by the firm [6]. In fact, weather risk also exists widely in many other industries, such as energy, agriculture, outdoor sports, and service business. As the abovementioned examples, there is a significant challenge for the retailer (newsvendor) to match their inventory levels with uncertain market demand which is sensitive to noncatastrophic weather conditions. 
In this paper, we try to address the joint decisions of inventory level and retail price in a newsvendor setting, where the newsvendor (retailer) sells a seasonal product and faces inventory risk due to weather uncertainty. (For more details about single-product models that deal with joint ordering and pricing issues, the reader is referred to [7] for a comprehensive review.) We develop a basic model with weather-sensitive demand. In our model, the risk-neutral retailer needs to jointly determine the retail price and the ordering quantity. Our model is intended to examine the following questions.

(1) How should the retailer determine the optimal retail price and order quantity for seasonal product under weather-sensitive demand?

(2) How would the optimal retail price and order quantity change as the model parameters (such as weather index (temperature), inventory holding cost) change?

In order to address the above questions and gain managerial insights into pricing and ordering policies for seasonal products, we need to model the uncertain market demand. Similar to [8] and some other studies in the literature (e.g., $[1,9]$ ), we model the market with a single representative customer whose valuation for the product is dependent on the weather condition. Without loss of generality, we assume that the customer's valuations are uniformly distributed. Furthermore, we develop a demand model, based on a customer's purchase probabilities, which is consistent with some articles, for example, Gallegos and Sahin [10]. In this paper, we extend the model by Gao et al. [3] in two directions: first, to investigate the joint optimal pricing and ordering decisions when demand is weather sensitive and second, to consider the customer's purchase behavior. Our works also differ from Demirag [8] by considering that the seasonal retailer price is endogenous. Our model mainly examines two issues (i.e., retail price and order quantity) under weathersensitive demand uncertainty that need to be addressed prior to the selling season. However, our model does not deal with the dynamic pricing issue that may occur during the selling season.

The rest of the paper is organized as follows. In the next section, we give a brief literature review, which is followed by the formulation of this problem. In Section 3, we first describe the basic model setting and state our assumptions (Section 3.1). We then proceed to the analysis of main model and results in Section 3.2. In Section 4, we present numerical examples to provide some managerial insights. Finally, we discuss some implications of our findings and suggest topics for future research in Section 5.

\section{Literature Review}

In this paper, our work is closely related to the literature in demand risk (weather risk caused by weather) management and joint ordering and pricing decisions of products. We briefly review the most relevant work to clarify the contribution of the paper.
There are many ways in which retailers can mitigate the impact of the demand uncertainty (caused by weather, price, and other factors) on their overall profit. The most common mechanisms involve operational hedging, which can be achieved via delayed product differentiation [11], quick response using more flexible subcontractor (or production) capacity [12], resource diversification and sharing [13], and the usual in-season and end-of-season markdowns, among others. For a more comprehensive discussion of operational hedging, see Boyabatli and Toktay [14]. Other works that consider demand uncertainty risk include $[15,16]$. The papers mentioned above do not involve weather-related demand uncertainty. However, many business firms may have to face the non-catastrophic weather conditions; the demand of seasonal products is influenced not only by inherent randomness but also by the weather during the selling season.

Our work is more closely related to weather risk management. The weather risk we discussed in this paper is receiving discussion from other literature. Sabir et al. [17] study the adverse weather through changes in the speed of individuals' car trips based on the entire commuting trip. $\mathrm{Hu}$ et al. [18] use integrated assessment models that combine geophysics and economics features to evaluate and compare global warming policies. Nikolopoulos and Fildes [19] consider the uncertainty that comes with short-term weather forecasts and study the exact effect of weather on weekly or daily sales, which has been given very little attention in the forecasting literature. Pfeil and Balakrishnan [20] think that convective weather is responsible for large delays and widespread disruptions in the U.S. National Airspace System, especially during summer. They demonstrate how raw convective weather forecasts, which provide deterministic predictions of the vertically integrated liquid, can be translated into probabilistic forecasts of whether or not a terminal area route will be blocked. Dos Santos [21] explores postgraduate consumers' attitude towards global warming in South Africa and shows that the respondents' attitude towards global warming which is within the context of derived demand is significant since consumers are indirectly responsible for businesses' greenhouse gas emissions. Ziser et al. [22] studies the effect of weather conditions on forecast accuracy and presents an improved methodology for the consideration of weather uncertainty in electricity demand forecasts. For a more comprehensive discussion of weather risk hedging, see Regnier [23]. Different from these papers, although we consider weather risk, we mainly focus on the joint pricing and ordering decision of seasonal products under weather-related demand uncertainty.

In the operations management literature, our work is related to the optimal pricing and ordering decisions of products in a newsvendor setting. There is abundant literature on pricing and ordering strategies of common products. Weng [24] develops optimal pricing and ordering strategies in a manufacturing and distribution system consisting of one manufacturer and one distributor. The system operates to meet price-sensitive random demand. Federgruen and Heching [25] address the simultaneous determination of pricing and inventory replenishment strategies for one firm facing demand uncertainty. They characterize the structure of an 
optimal combined pricing and inventory strategy. Chen and Bell [26] address the simultaneous determination of price and inventory replenishment when customers return products to the firm. They derive results for the optimal prices and order quantities and discuss how the firm should change price and inventory quantities in order to mitigate the negative effects of returns from customers. Jia [27] analyzes production capacity competition among manufacturers and ordering quantities and pricing competitions among retailers in a supply chain network. The combined pricing and ordering decisions of a product with different brands are also considered. Jia and Zhang [28] consider the problem of dynamic pricing and ordering decisions for a kind of durable products with multiple generations in a supply chain with one manufacturer and one retailer. They show that the retailer's optimal ordering and pricing strategy exist and only depend on the consumer's quality and price sensitivities for a given product quality. Wei et al. [29] study a two-stage game problem on pricing, ordering, and allocation in a service supply chain, where one supplier sells a product with a fixed capacity to customers via two retailers under wholesale price contracts. Other works that consider the newsvendor problem include [3033]. However, to the best of our knowledge, no studies have been conducted on the joint pricing and ordering decisions under weather and price sensitivities demand.

Our work is most closely related to Gao et al. [3] and Demirag [8]. Gao et al. consider the problem of hedging inventory risk for a seasonal product whose demand is sensitive to weather conditions. Our work differs from Gao et al. [3] in the following aspects. First, our random demand model is based on a customer's purchase probabilities, whereas they assume the seasonal demand is stochastically decreasing in weather index. Second, we investigate the optimal pricing and ordering decisions of seasonal products while they study the joint hedging and ordering decisions. Demirag analyzes the performance of weather-conditional rebates by explicitly considering the impact of different risk behaviors. Our model differs from Demirag [8] in the following manner. Specifically, the retail price is a decision variable, whereas they do not consider the pricing issue for seasonal product during the regular selling season.

\section{The Basic Model}

3.1. Problem Description and Assumptions. In this study, our objective is to investigate the joint optimal pricing and ordering decisions of seasonal products. Particularly, we consider a risk-neutral retailer that sells a seasonal product with weather-sensitive demand. The demand occurs during the regular selling season and is contingent on the seasonal weather status. In this paper, we represent the seasonal weather status with the average seasonal temperature $w$ which is uncertain and unknown prior to selling season. We assume that $w$ can be in two possible states, namely, "High" and "Low", with probabilities $\alpha$ and $\bar{\alpha}=1-\alpha$, where $w$ is above and below a specified threshold level $\bar{w}$, respectively. Depending on the characteristics of the product, different values of $w$ lead to more or less "favorable" conditions for its use, and customers' valuations determine the sales under different weather states. For example, the retailer can expect to generate higher sales of cooling appliances during a "hot" summer.

Next, we proceed to discuss our assumptions. Without loss of generality, we assume that lower values of $w$ represent more favorable conditions and assume the customer's valuation is stochastically decreasing with $w$. The weather state is revealed at the beginning of the regular selling season. Subsequently, the customers can purchase at the prices $p^{L}$ (in the low temperature state) or $p^{H}$ (in the high temperature state) depending on the realized temperature state. The seasonal retail prices $p^{L}$ and $p^{H}$ are endogenous and known prior to the promotional period. Note that our model is different from that of $[8,9]$, which assumes that the seasonal retail prices are exogenous.

At the beginning of the selling season, the retailer only knows the distribution of the average temperature $w$. The retailer makes inventory decisions and also determines the seasonal retail prices. In particular, the retailer can order seasonal products at a per-unit purchasing cost of $c$ and incurs a unit inventory holding cost of $h$. There is no fixed cost for placing an order; therefore, the ordering cost mainly depends on the wholesale price charged by the supplier. After the weather state revealed, we assume that the retailer can place a second order at a per-unit purchasing cost of $\widetilde{c}$, which can be called emergency ordering or quick response strategies used in practice. Each unit of surplus products can be salvaged at a value of $s$.

Similar to $[8,9]$ and some other studies in the literature (e.g., [34, 35]), we model the market demand using a single representative customer whose valuation for the product is dependent on the temperature. The retailer does not know the customer's valuation but associates a probability distribution for the possible values. In particular, corresponding to the two temperature states of high and low, we assume that two respective customer valuations $v^{H}$ and $v^{L}$ are uniformly distributed in $\left(0, \widetilde{v}^{H}\right)$ and $\left(0, \widetilde{v}^{L}\right)$, where $\widetilde{v}^{H}$ and $\widetilde{v}^{L}$ are the maximum valuations of the customer in the high and low states, respectively, and $\widetilde{v}^{H}<\widetilde{v}^{L}$. For ease of tractability of analysis, we assume that valuation distributions for the two states are independent. In this paper, the demand model is based on the customer's purchase probabilities, which is consistent with some articles, for example, Gao et al. [9] and Gallego and Sahin [10]. We also note that characterizing the market with a single representative customer has been used in several other studies, for example, Demirag [8] and Chen et al. [34]. The customer's higher average valuation in a low temperature state can be justified based on the product characteristics; for example, the customer's valuation on a new-style down jacket is expected to be higher during a chilly winter (low temperature state) than a warm winter (high temperature state). Our analysis can be easily modified to handle the opposite cases; for example, a customer's valuation on an air conditioner is expected to be higher during a hot summer than that during a cool summer.

To rule out the uninteresting or trivial cases, we assume that the following relationships exist among the various 


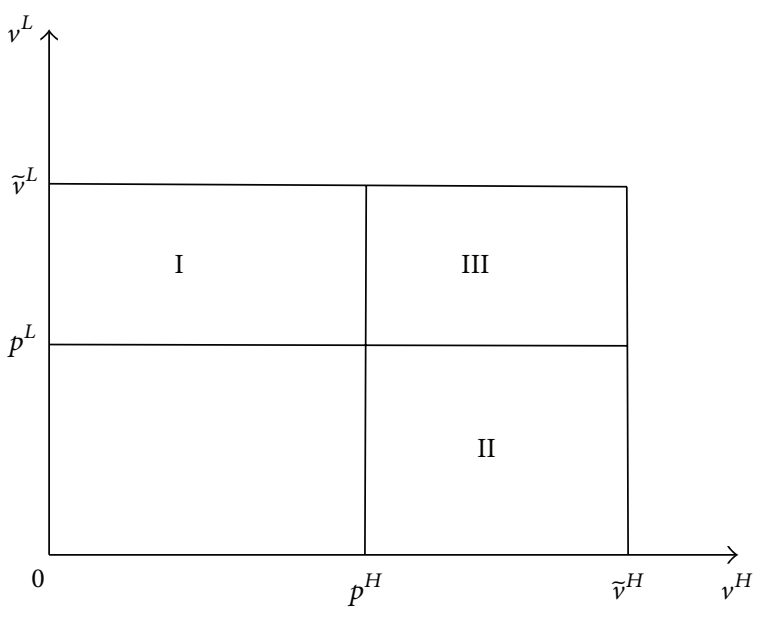

FIGURE 1: Demand corresponding to the two temperature states.

model parameters: $p^{L}<v^{L}, p^{H}<v^{H}, s<c<p^{L}$, and $s<c<p^{H}$. The key notations used in this paper are summarized in Notation section.

We now turn out our attention to the main model and provide a technical analysis of the main problem.

3.2. Main Model and Analysis. In our analysis, the retailer first determines the optimal order quantity under market demand uncertainty. Then, the retailer determines the optimal selling price $p^{L}$ and $p^{H}$ and sells the seasonal products during the normal selling period. To analyze our main model, we characterize the demand corresponding to the two temperature states as follows.

When the temperature is high, that is to say, $w>\bar{w}$, the demand in this state, $D^{H}$, is given by

$$
D^{H}=\operatorname{Pr}\left(v^{H}>p^{H}\right)=\frac{\widetilde{v}^{H}-p^{H}}{\widetilde{v}^{H}},
$$

and when the temperature is low, that is to say, $w \leq \bar{w}$, the demand in this state, $D^{L}$, is given by

$$
D^{L}=\operatorname{Pr}\left(v^{L}>p^{L}\right)=\frac{\widetilde{v}^{L}-p^{L}}{\widetilde{v}^{L}} .
$$

Under both the cases, the representative customer makes a purchase if the selling price is lower than her valuation. From (1) and (2), we have two alternative scenarios depending on the relative size of $D^{H}$ and $D^{L}$. Now, we first analyze the case $D^{H} \leq D^{L}$ (the analogous result for the case $D^{H} \geq D^{L}$ can be easily obtained).

We illustrate an intuitive understanding of the demand in Figure 1. The horizontal axis stands for the customer's valuation in the high temperature state, and the vertical axis stands for the customer's valuation in the low temperature state. The area I + III is the demand $\left(D^{L}\right)$ when the temperature is low and the area II + III is the demand $\left(D^{H}\right)$ when the temperature is high. Note that the area III is the intersection of $D^{L}$ and $D^{H}$ corresponding to the case in which the customer's valuations justify purchases in both low and high temperature states. Therefore, the sum of I, II, and III in Figure 1 represents the demand, which is generated by the customer's purchases in one or both temperature states.

The retailer's order quantity decision in the selling period is denoted by $q$. Once the selling season starts, the retailer learns the demand state and makes an adjustment on the inventory; that is to say, if the demand is smaller than $q$, the surplus is salvaged at the per-unit value $s$; otherwise he can reorder to meet the excess demand completely at the per-unit emergency ordering cost $\tilde{c}$. In the latter, since the reordering decision is made after the realization of demand state is realized, the quantity to be reordered is not a decision variable.

The retailer's profit as a function of his order quantity decision, pricing decisions, and the realized weather state is as follows:

$$
\pi_{r}=\left\{\begin{aligned}
& p^{L} \min \left(D^{L}\left(p^{L}\right), q\right) \\
&+\left(p^{L}-\tilde{c}\right)\left(D^{L}\left(p^{L}\right)-q\right)^{+} \\
&+s\left(q-D^{L}\left(p^{L}\right)\right)^{+}-c q-h q \quad w \leq \bar{w} \\
& p^{H} \min \left(D^{H}\left(p^{H}\right), q\right) \\
&+\left(p^{H}-\widetilde{c}\right)\left(D^{H}\left(p^{H}\right)-q\right)^{+} \\
&+s\left(q-D^{H}\left(p^{H}\right)\right)^{+}-c q-h q \quad w>\bar{w} .
\end{aligned}\right.
$$

From (3),

$$
\pi_{r}=\left\{\begin{array}{cc}
(\widetilde{c}-c-h) q+\left(p^{L}-\widetilde{c}\right) D^{L}\left(p^{L}\right) & \\
-(\widetilde{c}-s)\left(q-D^{L}\left(p^{L}\right)\right)^{+} & w \leq \bar{w} \\
(\widetilde{c}-c-h) q+\left(p^{H}-\widetilde{c}\right) D^{H}\left(p^{H}\right) & \\
-(\widetilde{c}-s)\left(q-D^{H}\left(p^{H}\right)\right)^{+} & w>\bar{w}
\end{array}\right.
$$

where $(a)^{+}=\max \{a, 0\}$ and $D^{H}$ and $D^{L}$ are characterized by (1) and (2). The revenue is generated from sales and salvaged units, and a cost of $c+h$ is incurred for each unit of order quantity.

Next, for given $p^{L}$ and $p^{H}$, we first characterize the retailer's optimal order quantity decision $q^{*}$ that maximizes expected profited $E \pi_{r}$. The retailer's expected profited is written as follows:

$$
\begin{aligned}
E \pi_{r}= & (\widetilde{c}-c-h) q+\left[\bar{\alpha}\left(p^{L}-\tilde{c}\right) D^{L}+\alpha\left(p^{H}-\tilde{c}\right) D^{H}\right] \\
& -(\widetilde{c}-s)\left[\bar{\alpha}\left(q-D^{L}\right)^{+}+\alpha\left(q-D^{H}\right)^{+}\right] .
\end{aligned}
$$

From (5),

$$
E \pi_{r}= \begin{cases}(\widetilde{c}-c-h) q+\bar{\alpha}\left(p^{L}-\widetilde{c}\right) D^{L} & \\ +\alpha\left(p^{H}-\tilde{c}\right) D^{H} & q \leq D^{H} \\ (\bar{\alpha} \widetilde{c}-\alpha s-c-h) q+\bar{\alpha}\left(p^{L}-\widetilde{c}\right) D^{L} & \\ +\alpha\left(p^{H}-s\right) D^{H} & D^{H}<q \leq D^{L} \\ (s-c-h) q+\bar{\alpha}\left(p^{L}-s\right) D^{L} & \\ +\alpha\left(p^{H}-s\right) D^{H} & D^{L}<q .\end{cases}
$$

The optimization problem becomes maximizing a piecewise liner function. By analyzing the coefficients of the 
terms with $q$ in the above expressions, we can obtain the retailer's optimal order quantity $q^{*}$ and expected profit $E \pi_{r}^{*}$. Theorem 1 summarizes our results.

Theorem 1. For given $p^{L}$ and $p^{H}$, we can obtain the retailer's optimal order quantity and expected profited for the case of $D^{H} \leq D^{L}$ as follows.

(i) If $h>\widetilde{c}-c$, then $q^{*}=0$, and

$$
E \pi_{r}=\bar{\alpha}\left(p^{L}-\widetilde{c}\right) D^{L}\left(p^{L}\right)+\alpha\left(p^{H}-\widetilde{c}\right) D^{H}\left(p^{H}\right) .
$$

(ii) If $h=\widetilde{c}-c$, then $q^{*} \in\left[0, D^{H}\right]$, and

$$
E \pi_{r}=\bar{\alpha}\left(p^{L}-\tilde{c}\right) D^{L}\left(p^{L}\right)+\alpha\left(p^{H}-\tilde{c}\right) D^{H}\left(p^{H}\right) .
$$

(iii) If $\bar{\alpha} \widetilde{c}+\alpha s-c<h<\widetilde{c}-c$, then $q^{*}=D^{H}$, and

$$
E \pi_{r}=\bar{\alpha}\left(p^{L}-\widetilde{c}\right) D^{L}\left(p^{L}\right)+\left(\alpha p^{H}-\bar{\alpha} \widetilde{c}-c-h\right) D^{H}\left(p^{H}\right) .
$$

(iv) If $h=\bar{\alpha} \widetilde{c}+\alpha s-c$, then $q^{*} \in\left[D^{H}, D^{L}\right]$, and

$$
E \pi_{r}=\bar{\alpha}\left(p^{L}-\tilde{c}\right) D^{L}\left(p^{L}\right)+\alpha\left(p^{H}-s\right) D^{H}\left(p^{H}\right) .
$$

(v) If $h<\overline{\alpha \widetilde{c}}+\alpha s-c$, then $q^{*}=D^{L}$, and

$$
E \pi_{r}=\left(\bar{\alpha} p^{L}+\alpha s-c-h\right) D^{L}\left(p^{L}\right)+\alpha\left(p^{H}-s\right) D^{H}\left(p^{H}\right) .
$$

Proof. From (6), it is easy to see that Theorem 1 holds.

Note that, if $h>\widetilde{c}-c$, it is unprofitable to order; hence it is optimal for the retailer to order nothing. The relationship between $h$ and $\bar{\alpha} \widetilde{c}+\alpha s-c$ can be rewritten as the relationship between $\alpha(h+c-s)$ and $\bar{\alpha}(\widetilde{c}-h-c)$, which, respectively, correspond to the overage cost when the temperature state is high and the shortage cost when the temperature state is low. Consequently, the retailer's order quantity is high (low) when faced with a high shortage (overage) cost.

Now, the effort required to compute the optimal pricing policy depends on the shape of $E \pi_{r}^{*}$. However, as Theorem 1 demonstrates, $E \pi_{r}^{*}$ might have multiple points that satisfy the first order optimality condition, depending on the parameters of the problem. Theorem 2 summarizes our main results.

Theorem 2. The optimal pricing decisions under the scenario $D^{H} \leq D^{L}$ are given by

$$
\begin{aligned}
p^{L^{*}} & =\frac{\bar{v}^{L}+x}{2}, \\
p^{H^{*}} & =\frac{\bar{v}^{H}+y}{2},
\end{aligned}
$$

where

$$
\begin{gathered}
x=\min \left(\widetilde{c}, \frac{1}{\bar{\alpha}}(c+h-\alpha s)\right), \\
y= \begin{cases}\widetilde{c} & h \geq \widetilde{c}-c \\
\max \left(s, \frac{1}{\alpha}(c+h-\bar{\alpha} \widetilde{c})\right) & h<\widetilde{c}-c .\end{cases}
\end{gathered}
$$

Proof. From (1), (2), and (7), if $h \geq \widetilde{c}-c$, then we get the expected value of $\pi_{r}\left(p^{H}, p^{L}\right)$ as

$$
E \pi_{r}\left(p^{H}, p^{L}\right)=\bar{\alpha}\left(p^{L}-\tilde{c}\right) \frac{\bar{v}^{L}-p^{L}}{\bar{v}^{L}}+\alpha\left(p^{H}-\tilde{c}\right) \frac{\bar{v}^{H}-p^{H}}{\bar{v}^{H}} .
$$

From (14), the first order derivative of $E \pi_{r}\left(p^{H}, p^{L}\right)$ with regard to $p^{H}$ is

$$
\frac{\partial E \pi_{r}\left(p^{H}, p^{L}\right)}{\partial p^{H}}=\alpha\left(1-\frac{p^{H}}{\bar{v}^{H}}\right)-\alpha \frac{1}{\bar{v}^{H}}\left(p^{H}-\tilde{c}\right) ;
$$

in order to identify values of $p^{H}$ that can satisfy this first order optimality condition, the second order derivative is given as follows:

$$
\frac{\partial^{2} E \pi_{r}\left(p^{H}, p^{L}\right)}{\partial\left(p^{H}\right)^{2}}=-2 \alpha \frac{1}{\bar{v}^{H}}<0
$$

From (16), the expected value of $E \pi_{r}\left(p^{H}, p^{L}\right)$ is concave in $p^{H}$ (in fact, it is easy to see that the expected profit $E \pi_{r}$ is jointly concave in $p^{L}$ and $p^{H}\left(\right.$ since $\partial^{2} E \pi_{r}\left(p^{H}, p^{L}\right) / \partial\left(p^{H}\right)^{2}<$ $0, \partial^{2} E \pi_{r}\left(p^{H}, p^{L}\right) / \partial\left(p^{H}\right)^{2}<0$, and $\partial^{2} E \pi_{r}\left(p^{H}, p^{L}\right) / \partial p^{H} \partial p^{L}=$ 0 , we can get a negative definite Hessian matrix)). Therefore, $E \pi_{r}\left(p^{H}, p^{L}\right)$ reaches its maximum at the unique value of $p^{H^{*}}$ that satisfies

$$
\frac{\partial E \pi_{r}\left(p^{H}, p^{L}\right)}{\partial p^{H}}=\alpha\left(1-\frac{p^{H}}{\bar{v}^{H}}\right)-\alpha \frac{1}{\bar{v}^{H}}\left(p^{H}-\tilde{c}\right)=0 .
$$

Thus, from (17), we get

$$
p^{H^{*}}=\frac{\bar{v}^{H}+\widetilde{c}}{2}
$$

Similarly, from (1), (2), and (7), we obtain that $\pi_{r}\left(p^{H}, p^{L}\right)$ reaches its maximum at the unique value of $p^{L^{*}}$ that satisfies

$$
\frac{\partial E \pi_{r}\left(p^{H}, p^{L}\right)}{\partial p^{H}}=\bar{\alpha}\left(1-\frac{p^{L}}{\bar{v}^{L}}\right)-\bar{\alpha} \frac{1}{\bar{v}^{L}}\left(p^{L}-\tilde{c}\right)=0 .
$$

Thus, from (19), we get

$$
p^{L^{*}}=\frac{\bar{v}^{L}+\widetilde{c}}{2}
$$

The analogous optimal pricing decisions for the case $\bar{\alpha} \widetilde{c}+$ $\alpha s-c<h<\widetilde{c}-c$ can be obtained as follows:

$$
\begin{aligned}
& p^{H^{*}}=\frac{\bar{v}^{H}+(1 / \alpha)(c+h-\bar{\alpha} \widetilde{c})}{2}, \\
& p^{L^{*}}=\frac{\bar{v}^{L}+\widetilde{c}}{2} .
\end{aligned}
$$


The analogous optimal pricing decisions for the case $h=$ $\bar{\alpha} \widetilde{c}+\alpha s-c$ can be obtained as follows:

$$
\begin{aligned}
& p^{H^{*}}=\frac{\bar{v}^{H}+s}{2}, \\
& p^{L^{*}}=\frac{\bar{v}^{L}+\widetilde{c}}{2} .
\end{aligned}
$$

The analogous optimal pricing decision for the case $h<$ $\bar{\alpha} \widetilde{c}+\alpha s-c$ can be obtained as follows:

$$
\begin{aligned}
& p^{H^{*}}=\frac{\bar{v}^{H}+s}{2}, \\
& p^{L^{*}}=\frac{\bar{v}^{L}+(1 / \bar{\alpha})(c+h-\alpha s)}{2} .
\end{aligned}
$$

For convenience of notation, we define new variables $x$ and $y$. Let

$$
\begin{aligned}
& x=\min \left(\widetilde{c}, \frac{1}{\bar{\alpha}}(c+h-\alpha s)\right), \\
& y= \begin{cases}\widetilde{c} & h \geq \widetilde{c}-c \\
\max \left(s, \frac{1}{\alpha}(c+h-\bar{\alpha} \widetilde{c})\right) & h<\widetilde{c}-c .\end{cases}
\end{aligned}
$$

Thus, we show a simplified expression for the retailer's optimal pricing decisions as follows:

$$
\begin{aligned}
p^{L^{*}} & =\frac{\bar{v}^{L}+x}{2}, \\
p^{H^{*}} & =\frac{\bar{v}^{H}+y}{2} .
\end{aligned}
$$

Therefore, Theorem 1 is proved.

For convenience of notation, from (24), we show a simplified expression for the retailer's optimal expected profit corresponding to the case of $D^{H} \leq D^{L}$ as follows:

$$
E \pi_{r}^{*}\left(p^{H}, p^{L}\right)=\bar{\alpha} \frac{\left(\bar{v}^{L}-x\right)^{2}}{4 \bar{v}^{L}}+\alpha \frac{\left(\bar{v}^{H}-y\right)^{2}}{4 \bar{v}^{H}}
$$

Similarly, we also show a simplified expression for the demand function corresponding to the case of $D^{H} \leq D^{L}$ as follows:

$$
\begin{aligned}
D^{L} & =\frac{\bar{v}^{L}-x}{2 \bar{v}^{L}}, \\
D^{H} & =\frac{\bar{v}^{H}-y}{2 \bar{v}^{H}} .
\end{aligned}
$$

TABLE 1: Simulation parameters.

\begin{tabular}{lcccccccc}
\hline Variable & $\alpha$ & $s$ & $c$ & $\widetilde{c}$ & $h$ & $\bar{w}$ & $\tilde{v}^{H}$ & $\tilde{v}^{L}$ \\
\hline Value & 0.5 & 182 & 200 & 239 & 10 & -4.2 & 400 & 500 \\
\hline
\end{tabular}

TABLE 2: Effects of weather conditions.

\begin{tabular}{lcccccc}
\hline$\alpha$ & $q^{*}$ & $D^{H^{*}}$ & $D^{L^{*}}$ & $p^{H^{*}}$ & $p^{L^{*}}$ & $\pi_{r}^{*}$ \\
\hline 0.400 & 0.271 & 0.136 & 0.271 & 291.000 & 364.330 & 33.978 \\
0.450 & 0.267 & 0.136 & 0.267 & 291.000 & 366.450 & 32.984 \\
0.500 & 0.135 & 0.135 & 0.262 & 291.500 & 369.000 & 31.944 \\
0.550 & 0.133 & 0.133 & 0.261 & 293.140 & 369.500 & 31.029 \\
0.600 & 0.131 & 0.131 & 0.261 & 295.340 & 369.500 & 30.057 \\
\hline
\end{tabular}

Theorem 3. The optimal ordering decision under the scenario $D^{H} \leq D^{L}$ can be written as follows:

$$
q^{*}= \begin{cases}0 & h>\widetilde{c}-c \\ {\left[0, \frac{\bar{v}^{H}-y}{2 \bar{v}^{H}}\right]} & h=\widetilde{c}-c \\ \frac{\bar{v}^{H}-y}{2 \bar{v}^{H}} & \bar{\alpha} \widetilde{c}+\alpha s-c<h<\widetilde{c}-c \\ {\left[\frac{\bar{v}^{H}-x}{2 \bar{v}^{H}}, \frac{\bar{v}^{L}-y}{2 \bar{v}^{L}}\right]} & h=\bar{\alpha} \widetilde{c}+\alpha s-c \\ \frac{\bar{v}^{L}-x}{2 \bar{v}^{L}} & h<\bar{\alpha} \widetilde{c}+\alpha s-c .\end{cases}
$$

Proof. From Theorem 1, (27), we can easily see that Theorem 3 holds.

\section{Numerical Examples and Further Analysis}

In this section, we mainly focus on examining the impact of the parameters on the retailer's decisions and present numerical analysis of the joint ordering and pricing decisions. It will be valuable to generate further managerial insights about the impact of parameters such as the temperature state and those associated with the costs. For the numerical examples, we select the basic case parameterized in Table 1. When a particular model parameter is under consideration, its value will be specified in corresponding examples and will differ from the basic case. In the following sections (Sections 4.1-4.3), we present computational studies to provide insights on the implementation of the optimal pricing and ordering. We first analyze the effects of the weather condition.

4.1. Impact of Weather Condition. The optimal retail price in the high temperature state, $p^{H^{*}}$, increases as the probability of a high temperature state, $\alpha$, increases (Table 2 ). The result implies that the retailer's optimal decision is to set the higher price as the probability of a high temperature increases. The analogous observation for the optimal retail price in the low temperature state, $p^{L^{*}}$, can be explained similarly. That is, $p^{L^{*}}$ increases as the probability of a high temperature state 
TABLE 3: Effects of inventory holding cost.

\begin{tabular}{lcccc}
\hline$h$ & $q^{*}$ & $p^{H^{*}}$ & $p^{L^{*}}$ & $\pi_{r}^{*}$ \\
\hline 8 & 0.266 & 290.000 & 367.000 & 33.229 \\
9 & 0.264 & 290.000 & 368.000 & 32.687 \\
10 & 0.262 & 290.500 & 369.000 & 32.149 \\
11 & 0.261 & 291.500 & 369.500 & 31.615 \\
12 & 0.258 & 292.500 & 369.500 & 31.086 \\
\hline
\end{tabular}

TABLE 4: Effects of emergency ordering cost.

\begin{tabular}{lcccc}
\hline$\widetilde{c}$ & $q^{*}$ & $p^{H^{*}}$ & $p^{L^{*}}$ & $\pi_{r}^{*}$ \\
\hline 229.000 & 0.261 & 295.500 & 364.500 & 32.010 \\
234.000 & 0.266 & 293.000 & 367.000 & 32.000 \\
239.000 & 0.270 & 291.500 & 369.500 & 31.872 \\
244.000 & 0.273 & 291.000 & 370.000 & 31.745 \\
249.000 & 0.273 & 291.000 & 370.000 & 31.881 \\
\hline
\end{tabular}

increases. The optimal order quantity, $q^{*}$, decreases as the probability of high temperature state increases. It is reasonable to expect that the retailer will decrease order quantity to save ordering costs when the weather (temperature) related market demand, $D^{H^{*}}\left(D^{L^{*}}\right)$, decreases as the probability of high temperature state increases.

4.2. Impact of Inventory Holding Cost. The optimal order quantity, $q^{*}$, decreases as the per-unit inventory holding cost, $h$, increases (Table 3 ). We can provide the following intuition. In order to save the ordering and holding costs, the retailer's optimal decision is to decrease order quantity as the perunit inventory holding cost increases. The optimal retail price in the low temperature state, $p^{L^{*}}$, increases as the per-unit inventory holding cost increases. The analogous observation for the optimal retail price in the high temperature state, $p^{H^{*}}$, can be analyzed similarly.

4.3. Impact of Emergency Ordering Cost. The optimal order quantity, $q^{*}$, increases as the per-unit emergency purchasing cost, $\widetilde{c}$, increases (Table 4). The observation is due to that a higher emergency purchasing cost will increase the total ordering costs of retailer; hence the retailer's optimal decision is to increase first order quantity, $q^{*}$. The optimal retail price in the high temperature state, $p^{H^{*}}$, decreases as the per-unit emergency purchasing cost increases. However, the optimal retail price in the low temperature state, $p^{L^{*}}$, increases as the per-unit emergency purchasing cost increases. The result reveals different monotonicity properties of $p^{H^{*}}$ and $p^{L^{*}}$ in $\widetilde{c}$. When the per-unit inventory holding cost increases, the retailer's profit decreases.

\section{Conclusions and Future Research}

The market demand for seasonal products such as cooling/heating appliances (air conditioner), new-style jacket, and snow equipment is weather sensitive. Naturally, adverse weather conditions that negatively affect the use of these items will reduce the demand. Retailers selling seasonal products have to face a challenge in matching their order quantities with uncertainty market demand during the selling season. Under this scenario, the market demand is shaped by the weather state and customer's valuations. And then, we present a joint decisions model in a newsvendor setting, where a retailer sells the seasonal products at the weather-dependent prices. We provide a detailed analysis of the problems and characterizations of the optimal decisions when the retailer is risk-neutral. We find that the optimal solution to the retail price and order quantity exists and it is the one and only. What is more, some useful insights, which are based on reasonable assumptions, have been generated in our extensive simulation experiments.

Our paper extends the analysis in Gao et al. [3] by providing a random demand model that is based on a customer's purchase probabilities as well as by studying the joint optimal pricing and ordering decisions. Our model differs from Demirag [8]. In this paper, the seasonal retailer price is the decision variable, whereas the seasonal retailer price is exogenous in [8]. For future research, our study can be extended at several directions. For example, we can extend the model by considering the retailer that sells multiple seasonal products under weather-sensitive demand uncertainty. Our study has a limitation that the model is independent of the customer valuations in different temperature states. In an extension, we may assume that the valuation distributions are dependent. Besides, our model also can be extended by considering the loss-aversion preference of retailer.

\section{Notation}

$w$ : The average seasonal temperature

$\alpha$ : The probability of a low temperature state

$\bar{\alpha}: \quad$ The probability of a high temperature state

$\bar{w}$ : The strike average temperature

$p^{L}$ : Retail price per unit in the low temperature state

$p^{H}$ : Retail price per unit in the high temperature state

$c$ : Purchasing cost per unit

$h$ : Inventory holding cost per unit

$\widetilde{c}: \quad$ Emergency purchasing cost per unit

$s: \quad$ Net salvage value per unit

$\widetilde{v}^{H}$ : The maximum valuations of the customer in high temperature state

$\widetilde{v}^{L}$ : The maximum valuations of the customer in low temperature state

$D^{H}$ : The demand in high temperature state

$D^{L}$ : The demand in low temperature state

q: Order quantity.

\section{Conflict of Interests}

The authors declare that there is no conflict of interests regarding the publication of this paper. 


\section{Acknowledgments}

The authors thank the two anonymous referees for their valuable comments and suggestions, which helped improve this paper. This research was supported by the National Natural Science Foundation of China (Grant nos. 70972056, 11301570) and the Specialized Research Fund for the Doctoral Program of Higher Education of China (Grant no. 20120191110042).

\section{References}

[1] F. H. Y. Chen and C. A. Yano, "Improving supply chain performance and managing risk under weather-related demand uncertainty," Management Science, vol. 56, no. 8, pp. 1380-1397, 2010.

[2] L. Eaenest, "Same-store sales rise 2. 9\% in May," Los Angeles Times, June 2005, http://articles.latimes.com/2005/jun/03/ business/fi-retail3.

[3] F. Gao, F. H. Y. Chen, and X. Chao, "Joint optimal ordering and weather hedging decisions: mean-CVaR model," Flexible Services and Manufacturing Journal, vol. 23, no. 1, pp. 1-25, 2011.

[4] M. Malinow, Weather Risk Management, Palgrave, New York, NY, USA, 2002.

[5] J. O'Donnell, “Wrong' weather can foul up retailers' sales," USA Today, 1B, November 2007.

[6] Speedwell Weather Derivatives Ltd, "How a fleece jacket manufacturer uses a PUT to protect against a mild winter," 2007, http://www.weatherderivs.com/WDExamples.htm.

[7] J. Eliashberg and R. Steinberg, Marketing-Production Joint Decision Making in Handbook in Operations Research and Management Science: Marketing, Elsevier Science Publishers, Amsterdam, The Netherlands, 1993.

[8] O. C. Demirag, "Performance of weather-conditional rebates under different risk preferences," Omega, vol. 41, pp. 1053-1067, 2013.

[9] F. Gao, Y. H. F. Chen, and O. C. Demirag, "Early sales of seasonal products with weather-conditional rebates," Production and Operations Management, vol. 21, no. 4, pp. 778-794, 2012.

[10] G. Gallego and O. Sahin, "Revenue management with partially refundable fares," Operations Research, vol. 58, no. 4, pp. 817833, 2010.

[11] H. L. Lee and C. S. Tang, "Modelling the costs and benefits of delayed product differentiation," Management Science, vol. 43, no. 1, pp. 40-53, 1997.

[12] A. V. Iyer and M. E. Bergen, "Quick response in manufacturerretailer channels," Management Science, vol. 43, no. 4, pp. 559570, 1997.

[13] J. A. van Mieghem, "Risk mitigation in newsvendor networks: resource diversification, flexibility, sharing, and hedging," Management Science, vol. 53, no. 8, pp. 1269-1288, 2007.

[14] O. Boyabatli and L. B. Toktay, "Operational hedging: a review with discussion," Working Paper, Technology Management Department, INSEAD, Fontainebleau, France, 2004.

[15] S. S. Sana, "Price sensitive demand with random sales price-a newsboy problem," International Journal of Systems Science, vol. 43, no. 3, pp. 491-498, 2012.

[16] S. S. Sana, "An EOQ model for stochastic demand for limited capacity of own warehouse," Annals of Operations Research, 2013.

[17] M. Sabir, J. van Ommeren, M. Koetse, and P. Rietveld, "Adverse weather and commuting speed," Networks and Spatial Economics, vol. 11, no. 4, pp. 701-712, 2011.
[18] Z. Hu, J. Cao, and L. J. Hong, "Robust simulation of global warming policies using the DICE model," Management Science, vol. 58, no. 12, pp. 2190-2206, 2012.

[19] K. Nikolopoulos and R. Fildes, "Adjusting supply chain forecasts for short-term temperature estimates: a case study in a Brewing company," IMA Journal of Management Mathematics, vol. 24, no. 1, pp. 79-88, 2013.

[20] D. M. Pfeil and H. Balakrishnan, "Identification of robust terminal-area routes in convective weather," Transportation Science, vol. 46, no. 1, pp. 56-73, 2012.

[21] M. A. O. dos Santos, "South African postgraduate consumer's attitude towards global warming," African Journal of Business Management, vol. 5, no. 11, pp. 4215-4225, 2011.

[22] C. J. Ziser, Z. Y. Dong, and K. P. Wong, "Incorporating weather uncertainty in demand forecasts for electricity market planning," International Journal of Systems Science, vol. 43, no. 7, pp. 1336-1346, 2012.

[23] E. Regnier, "Doing something about the weather," Omega, vol. 36, no. 1, pp. 22-32, 2008.

[24] Z. K. Weng, "Pricing and ordering strategies in manufacturing and distribution alliances," IIE Transactions, vol. 29, no. 8, pp. 681-692, 1997.

[25] A. Federgruen and A. Heching, "Combined pricing and inventory control under uncertainty," Operations Research, vol. 47, no. 3, pp. 454-475, 1999.

[26] J. Chen and P. C. Bell, "The impact of customer returns on pricing and order decisions," European Journal of Operational Research, vol. 195, no. 1, pp. 280-295, 2009.

[27] J. Jia, "Study on the competition equilibrium problems in a supply chain network under make-to-order policy," Journal of System Engineering, vol. 24, no. 3, pp. 299-306, 2009.

[28] J. Jia and J. Zhang, "Dynamic ordering and pricing strategies in a two-tier multi-generation durable goods supply chain," International Journal of Production Economics, vol. 144, pp. 135142, 2013.

[29] Y. Wei, Q. Hu, and X. Chen, "Ordering, pricing and allocation in a service supply chain," International Journal of Production Economics, vol. 144, pp. 590-598, 2013.

[30] S. S. Sana, "The stochastic EOQ model with random sales price," Applied Mathematics and Computation, vol. 218, no. 2, pp. 239248, 2011.

[31] S. S. Sana and K. Chaudhuri, "A stochastic EOQ policy of colddrink-for a retailer," Vietnam Journal of Mathematics, vol. 33, no. 4, pp. 437-442, 2005.

[32] B. Pal, S. S. Sana, and K. Chaudhuri, "A distribution-free with nonlinear holding cost," International Journal of Systems Science, 2013.

[33] S. S. Sana, "An economic order quantity model for nonconforming quality products," Service Science, vol. 4, no. 4, pp. 331-348, 2012.

[34] Y. Chen, S. Moorthy, and Z. J. Zhang, "Research note-price discrimination after the purchase: rebates as state-dependent discounts," Management Science, vol. 51, no. 7, pp. 1131-1140, 2005.

[35] E. Gerstner and J. D. Hess, "A theory of channel price promotions," American Economic Review, vol. 81, no. 4, pp. 872-886, 1991. 


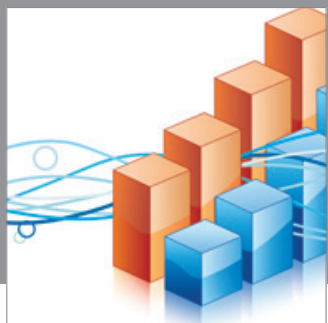

Advances in

Operations Research

mansans

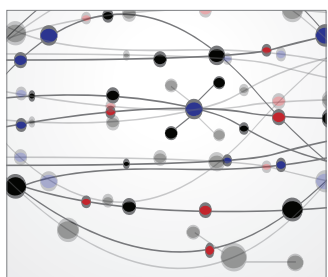

The Scientific World Journal
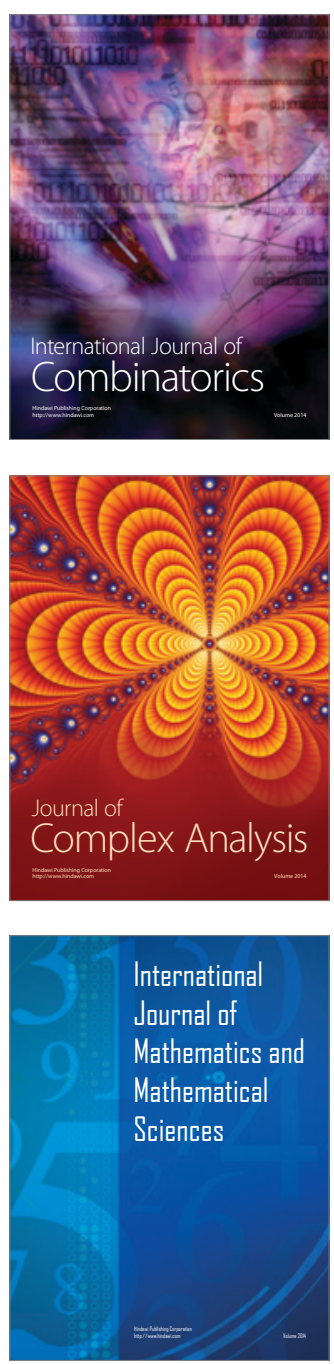
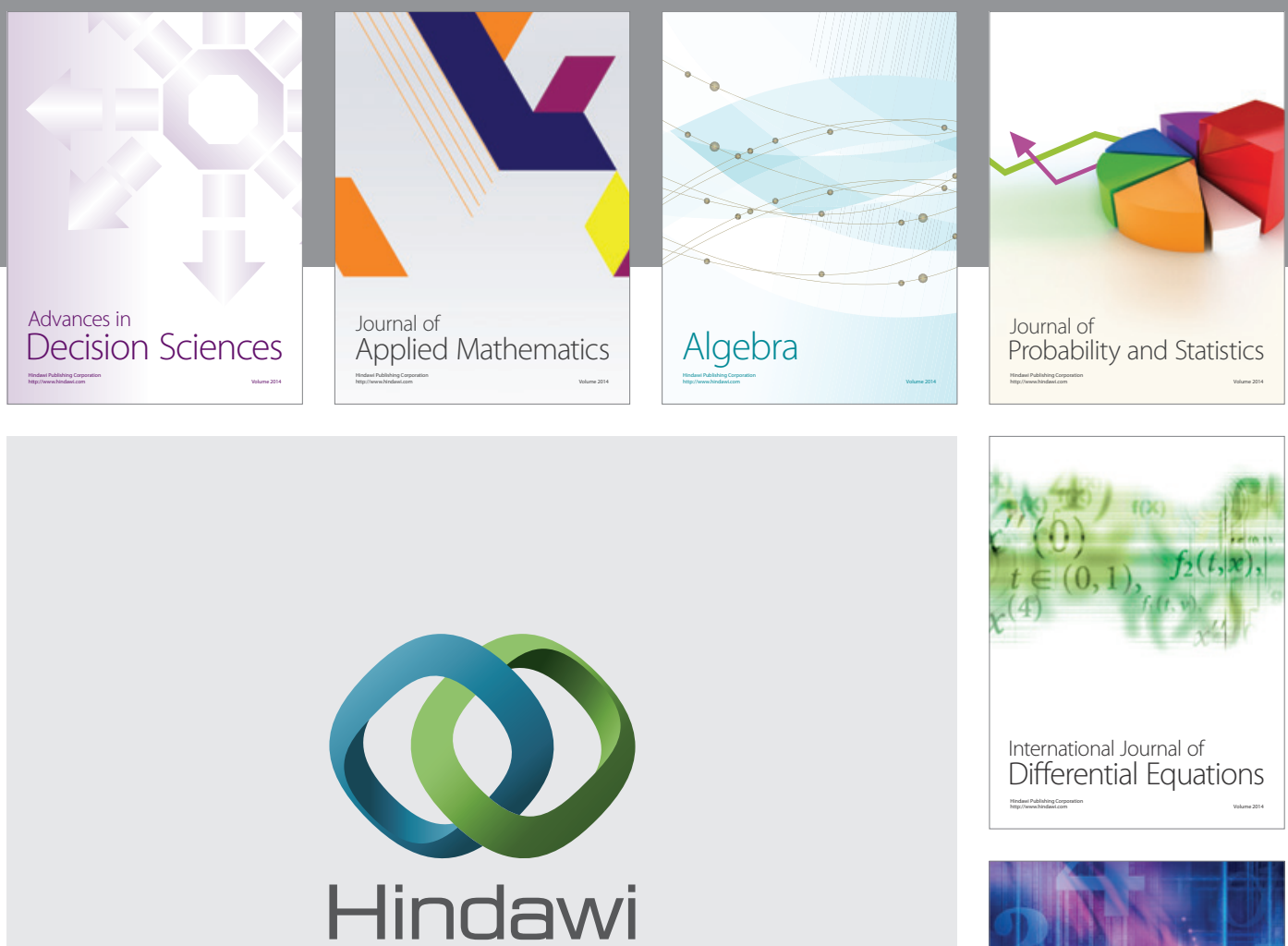

Submit your manuscripts at http://www.hindawi.com
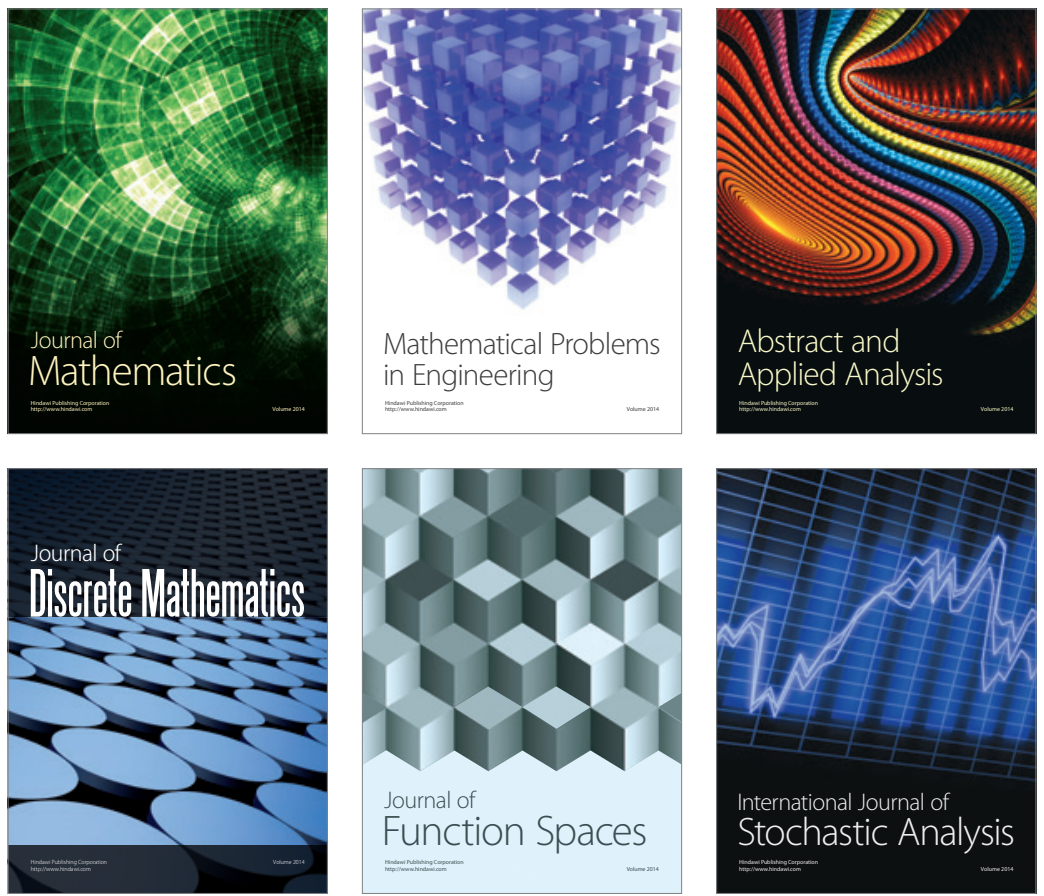

Journal of

Function Spaces

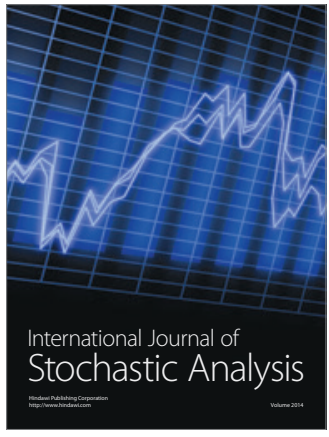

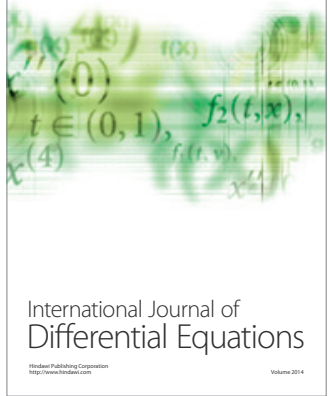
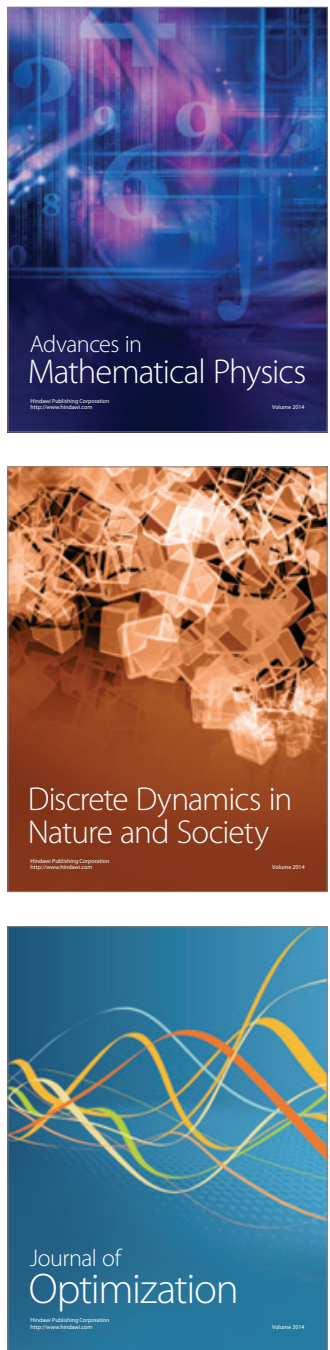